\title{
Shy/Silent Users of Contraceptives in Pakistan
}

\author{
SULTAN S. HASHMI \\ Based on the data of three national surveys, 1984-85 Pakistan Contraceptive \\ Prevalence Survey (PCPS), 1990-91 Pakistan Demographic and Health Survey (PDHS), and \\ 1994-95 Pakistan Contraceptive Prevalence Survey (PCPS), the hypothesis of shy/silent \\ users is tested. These surveys were undertaken with the collaboration of the Westing House, \\ IRD/Macro International and Local Office in Islamabad of the Population Council, New \\ York respectively. The concept of shy/silent users is defined as those respondents who, at the \\ time of interview, did not divulge that they were users of contraceptive methods or traditional \\ ways of preventing conception or birth due to cultural reasons. All three surveys show \\ substantial numbers of shy/silent users. If these numbers are included, the Current Prevalence \\ Rate (CPR) of each survey rises significantly. But the CPR inspite of including shy users, is \\ still far lower than most developing and neighbouring countries.
}

The concept of shy users of contraceptives was first developed on the basis of results of the Pakistan Contraceptive Prevalence Survey (CPS) of 1984-85. This survey was undertaken with the financial support of USAID, and Westing House, with the agreement of USAID, provided the technical support. The field work was carried out by female interviewers under the [Government of Pakistan (1986), pp. i-iii].

A hypothesis based on reports of some of the field staff was developed that there were respondents who were shy and reluctant to respond to questions on the use of contraceptives, especially when other family members were present at the time of interview. This hypothesis was tested by using the PCPS 1984-85 data and was further verified by using the data obtained, through the 1990-91 Pakistan Demographic and Health Survey (PDHS) [National Institute of Population Studies (1992)] and 1994-95 Pakistan Contraceptive Prevalence Survey (PCPS) [Government of Pakistan (1995)].

\section{SHY USERS IN PCPS, 1984-85}

Based on the data obtained through the PCPS 1984-85, binary technique was used and the results are shown in Figure 1 which are self explanatory. The data were continuously dichotomised until the number of those respondents (currently married women 15-49 years of age), who were fecund but had no births during the last five years preceding the survey was obtained.

Sultan S. Hashmi is Resident Adviser, National Institute of Population Studies, Islamabad.

Author's Note: Thanks are due to Syed Imtiaz Hussain Shah and Mohammad Sabir for typing various drafts of this paper. The views expressed are of the author alone. 
Fig. 1.

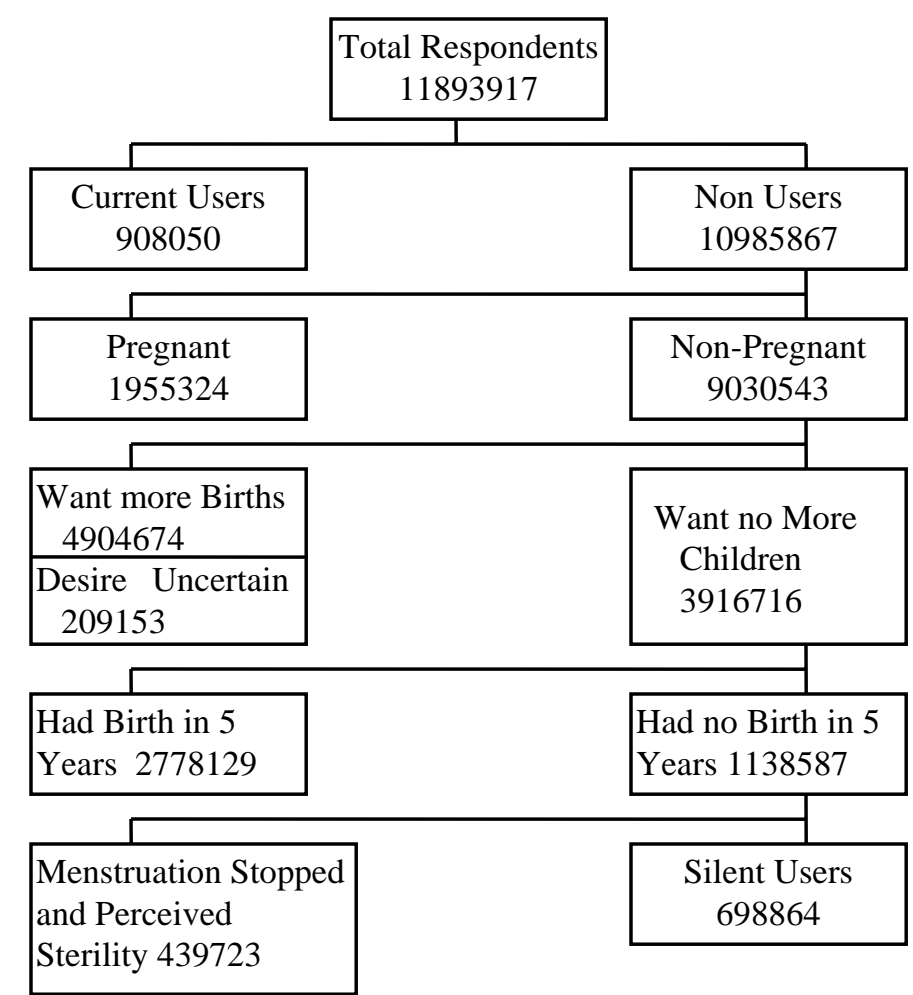

Source: PCPS, 1984-95.

Shy/silent users: 698,864.

Total users: $908,050+698,864=1,606,914$.

It is shown in Figure 1 that of the estimated 11,893,917, there were 698,864 or 5.9 percent respondents who were fecund and met all conditions to have births but they did not have any birth during the five year period preceding the survey. Also they did not report that they were users of contraceptives. Adding their number to those who reported that they were contraceptive users, the Contraceptive Prevalence Rate (CPR) for 1984-85 increased from 7.6 to 13.5 percent [Government of Pakistan (1986), pp. 86-87]. This level of CPR is obtained inspite of excluding those respondents whose desire for more births was uncertain. Besides, this revised level of CPR is more consistent with the observed level of TFR of 5.95 [Government of Pakistan (1986), p. 40]. 
Besides, it has been observed in several inquiries that women are shy to report methods especially, used by males. If women and their husbands are using traditional procedures, they do it to maintain confidentiality and some consider that since they are not using any modern method or material, it does not tantamount to contraceptive use.

\section{SHY USERS IN PDHS, 1990-91}

Similar, but more intensive analysis was undertaken of the data of 1990-91 Pakistan Demographic and Health Survey which provided an estimate of current CPR of 11.8 percent. The data obtained through this survey was further refined to determine the shy/silent users defined as currently married women 15-44 years of age, who had no births during the five or more years preceding the survey, were not sterile, had no menopause, were reported not using the contraceptive methods, had at least produced one live birth since marriage, were less than 45 years of age (all married women 45-49 years were excluded) and their ability to conceive had not been impaired.

In addition to the CPR of 11.8 percent the survey reported a total fertility rate (TFR) of 5.2 per woman for the year preceding the survey date and 5.4 prevailing over past six years preceding the survey. Although the reported CPR was low and TFRs were high by the standard of the world or of developing countries, the change over the previous survey of 1984-85 was viewed with skepticism. It was, therefore, hypothesised that the reported CPR was also under-reported due to shyness. Thus the main objective of the analysis of 1990-91 PDHS was to verify the hypothesis and to throw more light on the prevalence of shyness.

The shy/silent users from this survey were determined by using the same binary technique which was applied to 1984-85 PCPS data with further refinement. The results are presented in Figure 2. In order to determine the number of shy/silent users it was ensured that the respondents were fertile and had produced atleast one child, were under 45 years of age and were not experiencing primary or secondary sterility.

It is observed that respondents (currently married women 15-44 years) who had no birth during the last five years were not sterile or menopaused, were reportedly not using any contraceptive method and their ability to conceive was not impaired, were considered as shy/silent users.

It is observed from Figure 2 that the percentage of shy/silent users in the PDHS was 11.9 percent. This implies that the actual CPR in the PDHS could have been 23.7 percent. This revised level of CPR in the country as of 1990-91 was far lower than levels in several South and South Asian countries. For example, the reported level of CPR around the period 1987-1990 in South Asia was far higher than for Pakistan. It was reported 49 percent in India, 41 percent in Bangladesh, 52 percent in Sri Lanka, 50 percent in Indonesia, 36 percent in the Philippines and 53 percent in Vietnam. The latest levels in these countries with some fluctuation are even higher. 
Fig. 2. Determination of Shy/Silent Users of Contraceptive Methods Among Currently Married Women, PDHS 1990-91.

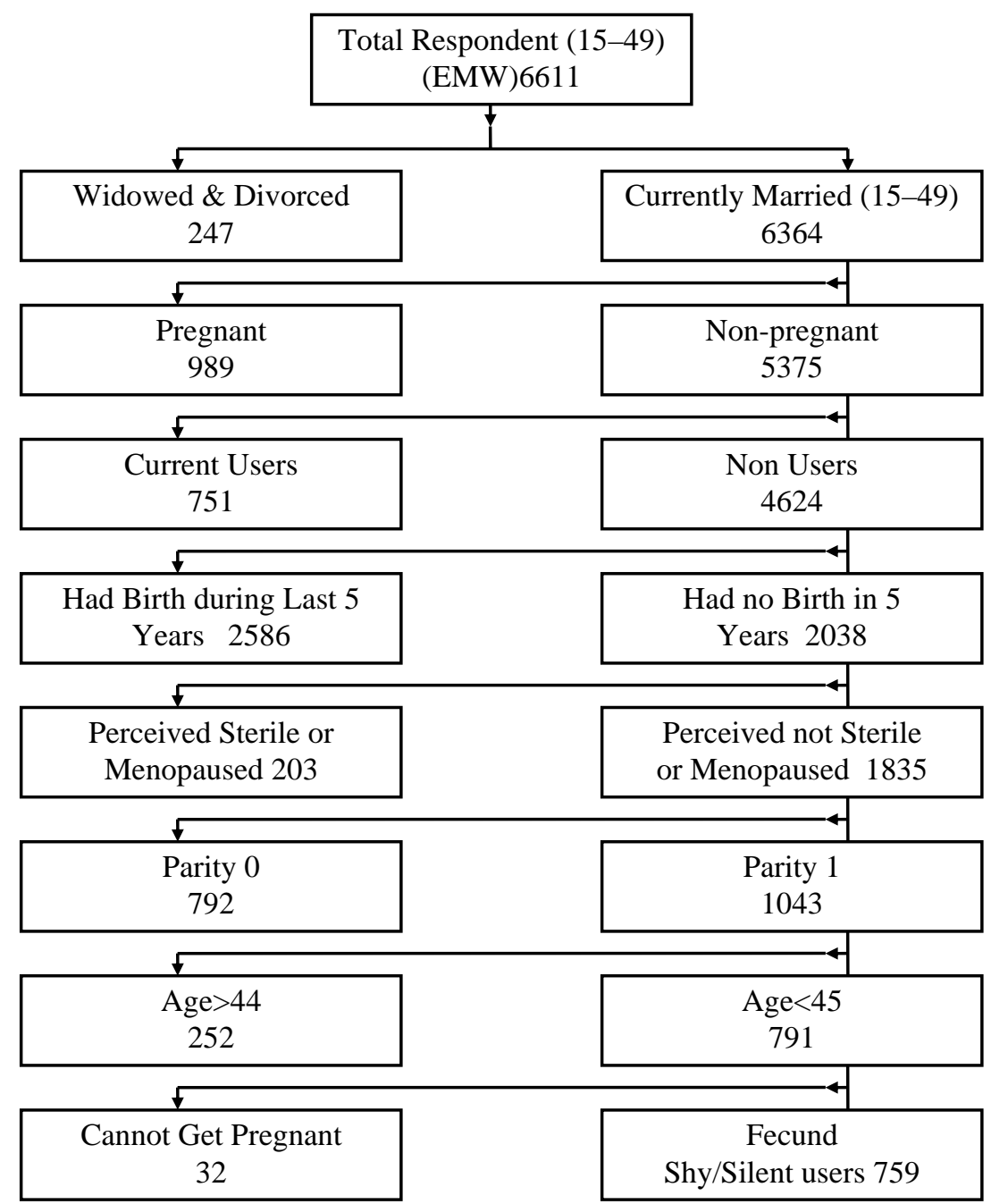

Source: PDHS 1990-1991.

Shy/Silent Users = 759 or 11.9 Percent of all Currently Married Women 15-49 Years of Age (6364); Reported Current Users = 751 or 11.8 Percent of all Currently Married 15-49 Years of Age; Total Users $=1510$ or 23.7 Percent of all Currently Married Women 15-49 Years of Age. 


\section{RELIABILITY OF TFR REPORTING VERSUS CPR AND FOLLOW-UP OF NON-USERS}

In view of cultural milieu it is pointed out that the reported TFR has been more dependable than the reported CPR, while there is a possibility that both these indicators might have been under-reported. The evidence is that birth reporting is more reliable than the reporting of CPR. The births which occurred during the past 12 months preceding the survey are less likely to be omitted as most of them ever born alive were present in the household and were, most probably in the lap of the mother being interviewed and could be seen by the interviewer. On the other hand the magnitude of omission of contraceptive use which is a personal matter depends on the subjectivity of the woman. It has been observed that many women in the country are culturally inhibited to talk about contraceptive use, especially, when stranger or member of the family, relatives and friends are present at the time of interview. Also there may be a self inhibition even if nobody except the interviewer is present. Thus, the CPR is under-reported due to shyness.

Another evidence to support the shy users comes from the follow-up survey of 844 non-users of the 1990-91 PDHS, which was undertaken during the first half of 1993. This follow-up was undertaken in Punjab province only. This survey (partly due

to the previous impact of the PDHS of 1990-91 ) showed a current prevalence rate of 18 percent of those who were reported as non-users in the 1990-91 PDHS.

The CPR reported in the 1990-91 PDHS for Punjab was 13 percent. In the follow-up survey the respondents reported a CPR of 18 percent. The 1993 method specific rates are compared with the total respondents of Punjab in the 1990-91 PDHS (Table 1).

It is noted that withdrawal was most used method (4.9 percent) followed by condom (4.4 percent) and the female's sterilisation (3.0 percent) while these rates reported for Punjab total in 1990-91 were, females sterilisation (3.8 percent), condom (3.0 percent) and withdrawal (1.5 percent) in that order. Apparently these were the users of traditional and male methods, who were more shy in the 1990-91 and who became more open in the 1993 follow-up survey.

This break through in a period of two years indicates that among other factors actual users of traditional and male methods were more shy to report at the time of PDHS in 1990-91 to admit that they were users and became more open in the followup inquiry.

\section{SHY USERS IN PCPS, 1994-95}

The 1994-95 Pakistan Contraceptive Prevalence Survey (PCPS) was funded by United Nations Population Fund (UNFPA) and was under-taken by the Population Council's local office Islamabad in coordination with the Ministry of Population 
Table 1

Percent Distribution of Follow Up Women 1993 and PDHS Married Women 19901991 of Age 15-49 Using Contraceptive Methods, Punjab Province

\begin{tabular}{clcc}
\hline S. No. & Contraceptive Methods & $\begin{array}{c}\text { PDHS(Punjab) } \\
1990-91\end{array}$ & FUW \\
\hline 0 & Any Method & 13.0 & 1993 \\
1 & Withdrawal & 1.5 & 4.9 \\
2 & Condom & 3.0 & 4.4 \\
3 & Female Sterilisation & 3.8 & 3.0 \\
4 & IUD & 1.5 & 1.7 \\
5 & Pill & 0.6 & 1.4 \\
6 & Injection & 0.8 & 0.9 \\
7 & Periodic Abstinence & 1.4 & 0.8 \\
8 & Other Methods & 0.3 & 0.8 \\
9 & Male Sterilisation & - & - \\
10 & Foam, Jell & - & - \\
& Not Currently Using & 87 & 82 \\
Percent & & 100 & 100 \\
Number & & 3768 & 844 \\
\hline
\end{tabular}

FUW= Follow up Women.

Welfare. This survey provides a CPR of 17.8 percent and as the data are still being processed, the TFR is still awaited. However, preliminary observations show that there were shy users of contraceptives in this survey who did not report that they were users.

A nationally representative sample of households and ever married women 15 to 49 years of age in these households were interviewed through this survey . The survey showed that there were 7922 currently married women 15-49 years of age. Out of these women there were 67 percent who had births during the period of last 59 months and 2567 had no births during the last 60 or more months.

Out of 2567 women who had no births there were 550 who never were pregnant, 274 had never a live birth, 484 were current users, 354 were menopaused, in case of another 13, husband was not present and the remaining 892 or 11.3 percent were considered as shy/silent users as they had no live birth nor they reported that they were using contraceptives during 60 or more months preceding the survey.

These figures are preliminary and subject to change when processing is checked and finalised. If this figure of shy users is added to the reported CPR, the percentage of current prevalence rate is increased to 29.1 percent. 


\section{Impact of Shy/Silent Users on CPRs}

All three surveys were undertaken in collaboration with the foreign agencies with the main objective of assessing the TFR and the CPR of the country. All three surveys were based on nationally representative samples. The interviewers in these surveys were well trained females who interviewed women respondents. The results obtained after the analysis of all three surveys show that the CPRs by including the shy/silent users increase substantially as shown in the following Table 2.

Table 2

Impact of Shy/Silent Users of Contraceptives on CPR of Pakistan Obtained in Surveys

\begin{tabular}{lcccc}
\hline Year of Survey & Survey & Reported Users & Shy Users & Total \\
\hline $1984-85$ & PCPS & 7.6 & 5.9 & 13.5 \\
$1990-91$ & PDHS & 11.8 & 11.9 & 23.7 \\
$1994-95$ & PCPS & 17.8 & 11.3 & 29.1 \\
\hline
\end{tabular}

The CPRs including the shy/silent users obtained through these surveys, especially, the first two, are consistent with the reported TFRs. The reported TFRs for the 1984-85 PCPS was 5.95 and for the 1990-91 PDHS was 5.2 for the 12 months preceding the survey per woman. The more detailed analysis of the 1994-95 PCPS could not be presented as the final processing of the data and analysis for obtaining TFR were awaited. Another noteable finding is that the percentage of shy/silent users went up from 5.9 percent in 1984-85 to 11.9 percent in 1990-91 and then it reduced to 11.3 percent in 1994-95.

\section{Characteristics of Shy/Silent Users}

The selected characteristic of shy/silent users as compared with the corresponding characteristics of reported current users have been gleaned from the 1990-91 PDHS [National Institute of Population Studies (1992), pp. 29-36]. These show that age composition of shy/silent users and the reported current users was slightly different. The average age of shy/silent users was 37.1 years as compared to 34.8 years of the current users. In other words shy/silent users were 2.3 years older than the current users. On the other hand in terms of average ages, husbands of shy/silent users were 37.1 years and of the reported current users were 36.8 years, meaning that the two groups were more or less similar. 
In terms of marriage half of shy/silent users had 20 or more years of duration while one third of reported current users had 20 or more years of duration.

With regard to education 83.5 percent of shy/silent users were illiterate while 52 percent of reported current users had no education. There were significant differences according to the place of residence as 70.9 percent of shy/silent users lived in rural areas whereas 34.1 percent of current users inhaled from rural setting.

There were significant differences between the two groups of users in respect of knowledge of atleast one contraceptive method which was 58.9 percent among the shy/silent users as compared to 88.5 percent of current users. While 26.2 percent of shy/silent users had the knowledge about the source of contraceptives, 64.3 percent of the reported users had this knowledge. On the other hand 83.7 percent of the shy/silent users reported that they never talked to their husbands about family planning, 25.3 percent of the reported current users said that they did not talk to their husbands.

It is argued that the users who are shy/silent users do not divulge the use of contraceptive especially, if other adults are present. The data show that in case of shy/silent users adults were present in 58.6 percent of the cases, while corresponding percentage for reported current users was 54.9 percent. Although the difference is small, it shows that this factor also contributed to the shyness.

With regard to consanguinity 51.7 percent of shy/silent users were married to their first cousin whereas 39.4 percent of reported current users were married to first cousin. More interesting is the number of children ever born to shy users which was 4.72 (slightly less) as compared to 4.99 CEB of current users.

These are a few characteristics of shy/silent users as compared to current users indicating that shy users are slightly older than the current users. Their husbands do not show much difference in ages, their marriage duration was longer. They were more illiterates. They lived predominantly in rural areas and had less knowledge of atleast one contraceptive method and source of contraceptive than reported current users. Most of them did not talk to their husbands about family planning and more were married to their first cousin. Because of shy/silent use, of probably traditional contraceptive methods, they had slightly fewer number of children. More information is presented in Table A given in the Appendix. 
Table A

Percentage Distribution of Shy/Silent Users and Current Users, Selected Characteristics, PDHS, 1990-91

\begin{tabular}{|c|c|c|}
\hline & Shy User & Current User \\
\hline \multicolumn{3}{|l|}{ Age of Respondent } \\
\hline$<20$ & - & 1.4 \\
\hline $20-24$ & 1.7 & 8.7 \\
\hline $25-29$ & 11.1 & 18.5 \\
\hline $30-34$ & 20.6 & 20.4 \\
\hline $35-39$ & 26.0 & 25.2 \\
\hline $40-44$ & 40.6 & 16.8 \\
\hline $45-49$ & - & 9.0 \\
\hline Total & 00.0 & 100.0 \\
\hline Average Age & 37.1 & 34.8 \\
\hline \multicolumn{3}{|l|}{ Age Difference between } \\
\hline \multicolumn{3}{|l|}{ Husband and Wife } \\
\hline Husband same Age or Younger & 9.2 & 9.2 \\
\hline Husband Older 1-2 Years & 9.9 & 14.0 \\
\hline " 3-4 " & 14.4 & 15.2 \\
\hline " 4-7 " & 29.4 & 24.8 \\
\hline " 8+ " & 37.1 & 36.8 \\
\hline \multicolumn{3}{|l|}{ Duration of Marriage } \\
\hline$<5$ Years & - & 5.6 \\
\hline 5-9 Years & 5.2 & 18.0 \\
\hline 10-14 Years & 20.0 & 21.7 \\
\hline 15-19 Years & 24.5 & 21.4 \\
\hline $20+$ Years & 50.3 & 33.4 \\
\hline \multicolumn{3}{|l|}{ Level of Education } \\
\hline No Education & 83.5 & 52.0 \\
\hline Primary & 7.0 & 13.5 \\
\hline Middle & 3.7 & 10.9 \\
\hline Secondary and Above & 5.8 & 23.6 \\
\hline
\end{tabular}


Table A-(Continued)

\section{Knowledge of At Least One \\ Contraceptive Method}

No

41.1

11.5

Yes

58.9

88.5

Knowledge about Source of

\section{Contraceptive}

No

73.8

35.7

Yes

26.2

64.3

\section{Talked to Husband about FP in the Past Year}

Never

83.7

25.3

One or Twice

12.2

33.4

More often

4.0

11.0

Sterilised

\section{Presence of Adults during}

Interview

No

41.4

45.1

Yes

\section{Relationship with Husband}

1st Cousin—Father Side

31.9

21.4

1st Cousin-Mother Side

19.8

18.0

2nd Cousin

10.1

12.9

Other Relatives

1.0

0.9

Non-relative

36.6

46.8

\section{No. of Children Ever Born}

$\begin{array}{lll}1-2 & 19.4 & 12.5 \\ 3-4 & 28.2 & 29.3 \\ 5-6 & 24.2 & 28.8 \\ 7+ & 28.2 & 29.2\end{array}$

Source: [Hashmi and Ahmad (1992)]. 


\section{REFERENCES}

Pakistan, Government of (1986) Pakistan Contraceptive Prevalence Survey, 1984-85. Islamabad: Population Welfare Division.

National Institute of Population Studies (1992) Pakistan Demographic and Health Survey, 1990-1991. Columbia, Maryland: IRD/Macro International Inc.

Pakistan, Government of (1995) Pakistan Contraceptive Prevalence Survey, 19944-95. Islamabad: The Population Council, The Ministry of Population Welfare.

Hashmi, Sultan S., and Tauseef Ahmad (1992) Shy/Silent Users of Contraception: Further Evidence. In Pakistan Population Review 3:1 Spring. 


\section{Comments}

The paper by Dr Hashmi seems to be a response to the general concern that after more than three decades of official and non-governmental programmes to promote widespread use of contraception for controlling fertility, the levels of contraceptive use prevalence as reported by various surveys are much lower than what was expected. His argument on the basis of the findings from a sequential binary procedure of analysis is that a notable proportion of the respondent women of reproductive ages, who were not reported as users, were in reality making efforts to avoid the occurrence of births, through the use of modern contraceptives or other means, but this was not reported to the survey interviewers either due to shyness or due to their considering such efforts to be not contraception. The hypothesis having emerged from the reports in this regard given by some interviewers of the 1984-85 PCPS, and due to the apparent inconsistency between the Total Fertility Rate and Contraceptive Prevalence Rate given by this survey, has been tested by the author from the data provided by three surveys; namely 1984-85 PCPS, 1990-91 PDHS and 1994-95 PCPS.

For 1984-85 PCPS the shy or silent users have been estimated as the residuals from the respondent currently married women, after sequentially separating the number of current users, the pregnant women, those wanting more births and those who were uncertain, those having birth in the last 5 years, and those having menopause or having secondary sterility, with the result that in addition to 7.6 percent reporting as users, another 5.9 percent were found to be logically silent users. Similar analysis of 1990-91 PDHS data included the sequential separation of the number of those who were reported as pregnant, current users, those having birth during the last 5 years, sterile or menopaused, with no birth (0 parity), those aged more than 44, and those who cannot get pregnant. This resulted in 11.9 percent women who should be logically shy or silent users, in addition to 11.8 percent already reported as current users. A follow up of 1990-91 PDHS non-users in Punjab provided validity to the inference of shy/silent users hypothesis.

Similarly from the 1994-95 PCPS it was construed that 11.3 percent of the currently married respondent women 11.3 percent in addition to 17.8 reported as users, were the shy/silent users.

The paper shows on the basis of the first two surveys, that the inclusion of shy users makes the percentage making efforts to limit fertility (including both the reported and the shy users) to be consistent with the TFR for the last 12 months.

This very interesting and useful paper has from more than one surveys demonstrated that the estimates of contraceptive users and contraceptive prevalence rates are not to be taken on the basis of what is reported directly, rather the important 
component of those who are making deliberate efforts for limiting their family size and are not reporting so, cannot be ignored because of their significantly high proportion. This also shows that the serious awareness of the population about the need to limit the family size is much more in the country than what is usually assumed. By implications the findings by Dr Hashmi also point toward the inadequacies of the survey procedures in eliciting data regarding female in general, which has also been demonstrated by many studies regarding the coverage of labour force participation. In their case the element of shyness should obviously be not as much as the one regarding the reproduction-related issues. In other words the element of shyness should not be wholly attributed to the respondent (which unfortunately is usually implied) rather the role of the interviewer (even if it is female), the short-time formal exposure for the interview (with little rapport or confidence), the stereotyped questionnaires and ways of asking questions, and many related factors come in the way of having reliable information.

Dr Hashmi's paper points to the need of checking the internal consistency, the reliability and validity of data collected from the surveys, even when better educated and trained interviewers are used. Since the sample size in such surveys is small, the quality of information collected from the respondents depends a lot on the skill of the data collectors and of the data analysts. The experience from the analysis of data collected from such surveys also shows that in terms of estimating parameters and details no one source is sufficiently reliable. One has to go into the details of each survey and also carefully look at the data from other surveys to be able to get more meaningful inferences.

I feel that the analysis should also have been extended to age groups, and by other characteristics so that an idea about the inhibitions in reporting may be assessed. Special enquiries in this respect may be made during the other field endeavours being made under the auspices of the NIPS.

Formerly of the

Mohammad Afzal

Pakistan Institute of Development Economics, Islamabad. 